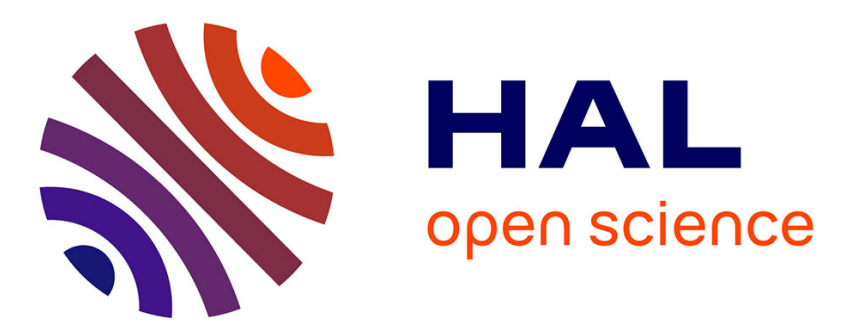

\title{
An EEG-fMRI Fusion Analysis Based on Symmetric Techniques Using Dempster Shafer Theory
}

\author{
Abdelouahab Attia, Abdelouahab Moussaoui, Youssef Chahir
}

\section{To cite this version:}

Abdelouahab Attia, Abdelouahab Moussaoui, Youssef Chahir. An EEG-fMRI Fusion Analysis Based on Symmetric Techniques Using Dempster Shafer Theory. Journal of Medical Imaging and Health Informatics, 2017, 7 (7), pp.1493-1501. 10.1166/jmihi.2017.2185 . hal-01621045

\section{HAL Id: hal-01621045}

\section{https://hal-normandie-univ.archives-ouvertes.fr/hal-01621045}

Submitted on 18 Sep 2018

HAL is a multi-disciplinary open access archive for the deposit and dissemination of scientific research documents, whether they are published or not. The documents may come from teaching and research institutions in France or abroad, or from public or private research centers.
L'archive ouverte pluridisciplinaire HAL, est destinée au dépôt et à la diffusion de documents scientifiques de niveau recherche, publiés ou non, émanant des établissements d'enseignement et de recherche français ou étrangers, des laboratoires publics ou privés. 


\title{
An EEG-fMRI fusion analysis based on symmetric techniques using Dempster Shafer theory
}

\author{
Abdelouahab ATTIA ${ }^{1}$,Abdelouahab MOUSSAOUI ${ }^{1}$,Youssef CHAHIR ${ }^{2}$ \\ Computer Science Department ,Faculty of Sciences \\ Ferhat Abbas University Setif I, Algeria \\ GREYC Laboratory - CNRS UMR 6072 \\ Departement of Computer Science \\ University of Caen Lower-Normandy-France \\ attia.abdelouahab@gmail.com
}

\begin{abstract}
EEG-fMRI data fusion provides a better insight of the brain activity due to its high spatiotemporal resolution. The current paper presents a new framework on EEG-fMRI data symmetric data fusion based on Dempster Shafer theory. Basically, symmetric methods require the use of a common theoretical model to explore and explain Electroencephalogram (EEG) and functional magnetic resonance imaging (fMRI) data jointly. Dempster Shafer theory has a multivariate use in resolving problems related to uncertainty. Accordingly, Basic Belief Assignment and the combination rule offered by such theory allow fusing multimodal sources such EEG (temporal modality) and fMRI (spatial modality). In particular, masse functions for each modality have been calculated. Then, the combination rule has been computed. Finally, this measure has been used to detect the activated areas in the brain via clustering using the potential-based hierarchical agglomerative clustering method. Both real auditory and artificial data simulation have been employed to evaluate the performance of the proposed approach. Also, true, false activation rates and Receiver Operating Characteristic (ROC curve) have been used to establish a comparison with jointICA method. The obtained results have clearly shown the ability of the introduced approach to outperform a standard method of data analysis to reveal a better activation map.
\end{abstract}

Keywords-; EEG-fMRI data fusion; symmetric as-symmetric approach; BOLD, HRF; Dempster Shafer theory; PHA method ;

\section{Introduction}

Electroencephalogram (EEG) is a non- invasive technique that measures the electrical activity of the brain [1]. It has gradually gained considerable attention of neuroscientists due to its high spatial resolution. Also, functional Magnetic Resonance Imaging (fMRI) [2] is a useful technique for measuring different attributes of the brain activity. Indeed, both of EEG and fMRI techniques have shown their efficiency in revealing a better insight of the brain activity.

Despite that, both EEG and fMRI modalities suffer from some limitations. On the one hand, fMRI data analysis relies on slow mechanisms such as blood flow, oxygen consumption and metabolism that yield only an indirect measure of the evoked BOLD (Blood-Oxygen-Level Dependent) signals. Given this, it is difficult to estimate the neural activity only from hemodynamic response. Also, it has been clearly observed that fMRI suffers from an ill-posed temporal inverse problem (i.e poor temporal resolution). On the other hand, the EEG modality suffers from spatial inverse problem besides noise raised due to the unavoidable artifacts during the course of the experiment that can strongly degrade the EEG signals quality [3] .

Both EEG and fMRI data measure neural activity. The EEG, revealing the event-related potentials (ERP) for investigating the psychophysiological states and information processing, is typical for the electrical activity. It is worth noticing that the evoked electromagnetic and metabolic responses detected by both EEG and fMRI are not essentially the result of the same primary neuronal processes [5].

Given that strengths and weaknesses of fMRI and EEG complete each other, simultaneous EEG-fMRI acquisition applications are fast becoming a key technique to provide a more comprehensive understanding of the nature of the cerebral activity with its enhanced spatiotemporal resolution [4]. Thus, several works have been recently developed involving the EEG/fMRI fusion. 
Mainly, the existing potential approaches devoted to develop EEG-fMRI integration procedures can be divided into two categories. In asymmetric methods, prior information of one modality is considered as a cause or a predictor of the second modality. In other word, it serves as a guide for the analysis of the other modality. Asymmetric approaches include: (i) fMRI informed EEG , known as fMRI-constrained EEG imaging, that employs spatial information from fMRI signal for reconstructing the source of the EEG signal [6] and (ii) EEG-informed fMRI technique that is concerned with modelling the fMRI signal with features from EEG convolved with the Hemodynamic Response Function (HRF) [7, 8]. EEG-fMRI symmetric methods are the second category of EEG-fMRI integration techniques. Mainly, the focus of these approaches is devoted to solving the inverse problem. They imply the use of a common forward or generative model to explore EEG and fMRI data jointly. Besides, they required some given knowledge from experience on the subject. $[9,10,11,12,13]$.

To fuse the EEG and fMRI sources, a framework proposed in [10] employs Bayesian theory to generate a common generative model. In this method, common parameters have been used to represent the spatial characteristics of both EEG and fMRI data. Therefore, the lack of detailed information about neurovascular coupling has not confused this fusion approach. Independent Component Analysis (ICA) has provided a powerful framework for integrating both modalities [6, 14]. To identify the component in EEG and fMRI independently, spatial ICA (sICA) for fMRI data and temporal ICA (tICA) for the EEG signal have been used. Due to the variation in spatial and temporal modalities, two methods have emerged: JointICA $[15,16]$ and parallel-ICA algorthim [17]. Another similar approach is a parallel framework for Spatial-Temporal EEG/fMRI Fusion (STEFF) [6]. Typically, STEFF has used an Empirical Bayesian (EB) model to combine temporal components from EEG and spatial components from fMRI obtained by ICA technique. Besides providing a mechanism for adjusting the problem of ignorance in ICA, STEFF has shown its ability to yield a flexible tool for imaging the EEG sources using information from fMRI data. For further detail, the reader is invited to see [ 18, 19,20]). Also, Dynamic Causal Model (DCM) is another framework for models of bioelectric and metabolic activity in neuronal populations $[21,22]$.

It is worth noticing that any symmetric fusion confronts three main problems: (i) The complexity of real metabolic-hemodynamic that renders the estimation of model-driven fusion problematic [11], (ii) The common substrate underlying EEG and fMRI signals should be identified by the fusion method and (iii) the uncertainty must be reduced by the fusion method when inferring this common subspace [10]. Given this, an extensive research has been conducted on EEG-fMRI fusion data analysis. However, EEG-fMRI fusion requires a common theoretical framework to analyze and combine information sources with different natures (modalities).

To enhance the accuracy of the combined EEG-fMRI analysis, the current work introduces a new approach based on symmetric fusion using Dempster Shafer evidence theory (DS). Basic Belief function and combination rule have been employed as major keys in the proposed method. Mainly, DS theory permits to study different data like EEG and fMRI because the combination rule measures enable to join these data. BOLD signal is usually considered to have the same time evolution as the EEG [12]. Given this, the HRF model permits analyzing fMRI and EEG signals in simultaneous EEG-fMRI data. Thus, in this approach, the HRF model has been used as a key element $[23,24,25,26]$ for modelling both EEG and fMRI modalities by DS theory to fuse spatiotemporal information. To detect activated areas in the brain, the Potential-Based Hierarchical Agglomerative (PHA) [27] have been employed. Therefore, PHA clustering method has time complexity of $\mathrm{O}\left(\mathrm{N}^{2}\right)$ and allows to choose the max number of clusters, denoted as (C). PHA generates automatically a number of clusters that is less than or equal to the selected max number (C). Among these clusters, we have shown the target cluster and more information like the number of voxels by cluster and the centroid of cluster denoted by (c).

The remaining paper is organized as follows: Section two describes the basics of Dempster Shafer theory. Besides the brief review of the hemodynamic response model, the proposed model by using Dempster Shafer is introduced in section three. Section four gives details on the proposed approach. The conducted experiments on real and simulation data are discussed with the various findings in section five. At last, a conclusion is given.

\section{Background of the Dempster-Shafer evidence theory}

Dempster-Shafer evidence theory has shown its efficiency in solving real problems [28]. It has been widely used in medical imaging and signal processing like prediction, classification, 
segmentation and information fusion [29,30]. In this work, two basic rules of DS theory are required: (i) Basic Belief Assignment (BBA) [31,32], (ii) Belief function, bel (.) and (iii) Dempster rule of combination. Thus, these fundamentals are described respectively as follows:

Definition1: Basic Belief Assignment (BBA) is a key point of evidence theory, referred to as $\mathrm{m}($.$) , and has the following form:$

$$
\mathrm{m}(\varnothing)=0 \text {, and } \sum_{\mathrm{Ai} \subseteq \ominus} \mathrm{m}(\mathrm{Ai})=1
$$

Definition 2: Belief function, bel (.).Let $\theta=\left\{\theta_{1}, \theta_{2}, \ldots, \theta_{\mathrm{k}}\right\}$ be a finite set of possible hypothesis. This set refers to the frame of discernment, and its power set is denoted by 2 where:

$$
2^{\theta}=\left\{\varnothing,\left\{\theta_{1}\right\},\left\{\theta_{2}\right\}, \ldots,\left\{\theta_{\mathrm{k}}\right\},\left\{\theta_{1} \cup \theta_{2}\right\},\left\{\theta_{1} \cup \theta_{3}\right\}, \ldots . \theta\right\}
$$

bel (.) is described as:

$$
\operatorname{bel}(\mathrm{Ai})=\sum_{\mathrm{Ai} \subseteq \mathrm{D}} \mathrm{m}(\mathrm{Ai})
$$

DS theory provides the combination rule that join several masses functions in one single masse probability of all modalities. The focus of this work has been also placed on employing the combined rule for pooling of evidence from two belief functions, Bel1 and Bel2

We assume two sources of evidence: $\mathrm{B}$ for EEG and $\mathrm{C}$ for fMRI are represented by respective subsets of $\theta: B_{1}, \ldots, B_{m}$ and $C_{1}, \ldots, C_{n}$. Probability density functions $m_{1}$ and $m_{2}$ are defined on $B$ and $C$ respectively. The Dempster rule of combination for combining two sets of masses $m_{1}$ and $m_{2}$ is defined as: $\mathrm{m}_{12}(\varnothing)=0$. A new value $\mathrm{m}_{12}(A)$ is calculated for each subset of $\theta$ as follows:

$$
\mathrm{m}_{12}(\mathrm{~A})=\frac{1}{1-\mathrm{k}} \sum_{\mathrm{B} \cap \mathrm{C}=\mathrm{A} \neq \varnothing} \mathrm{m}_{1}(\mathrm{~B}) \mathrm{m}_{2}(\mathrm{C})
$$

where $\mathrm{k}$ denotes a measure of the amount of conflict between two evidences. If $\mathrm{k}=1$ the two evidences cannot be combined because their cores are disjoint. This rule is commutative, associative, but not idempotent or continuous. $\mathrm{k}$ is calculated as follows:

$$
\mathrm{k}=\sum_{\mathrm{B} \cap \mathrm{C}=\varnothing} \mathrm{m}_{1}(\mathrm{~B}) \mathrm{m}_{2}(\mathrm{C})
$$

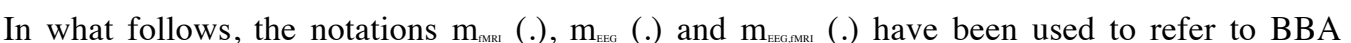
(fMRI), BBA (EEG) and the rule of combination respectively.

\section{Modelling HRF by Dempster Shafer method}

The canonical model proposed in [28] is one of the numerous HRF models that have been developed in literature. It has a vital role to play in characterizing the onset of the stimulus. This work focuses on the study of the so-called sum two gamma functions [33]. The first is for modelling the peak whereas the second is for modelling undershoots. The HRF model that is typical for BOLD impulse response is presented in Figure 1.

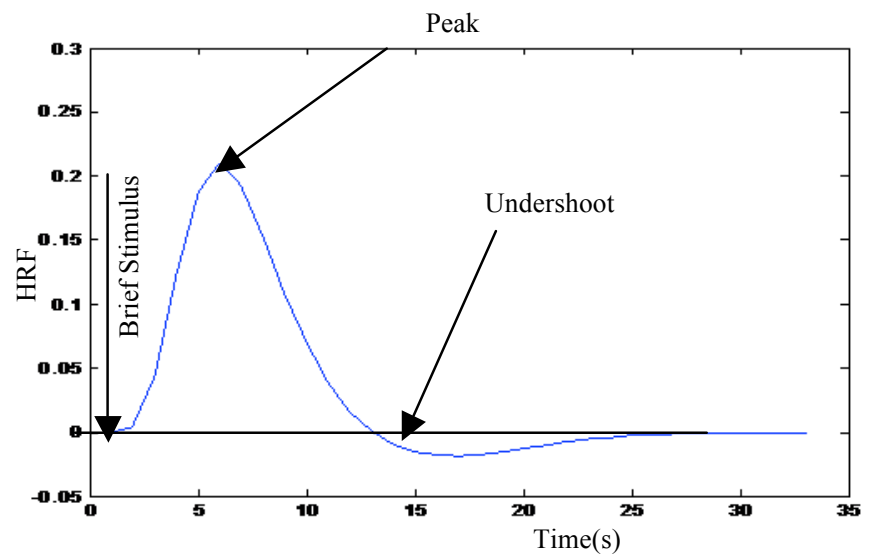


Figure 1. Canonical hemodynamic response function model

To extract more information about the activated areas, the HRF function has been modelled as follows: First, HRF function has been divided into two hypotheses $\left(\theta_{i}, \theta_{j}\right) . \theta_{i}$ corresponds to modelling the neural activation and determining a peak (on activation). $\theta_{j}$ is assigned to modelling the undershoot (off activation). In particular, the first hypothesis is the focus of the current work. It is divided into two parts A and D where A stands for degrees of belief included in D. In other words, (D minus A) stands for uncertainty. Figure 2 depicts the proposed model.

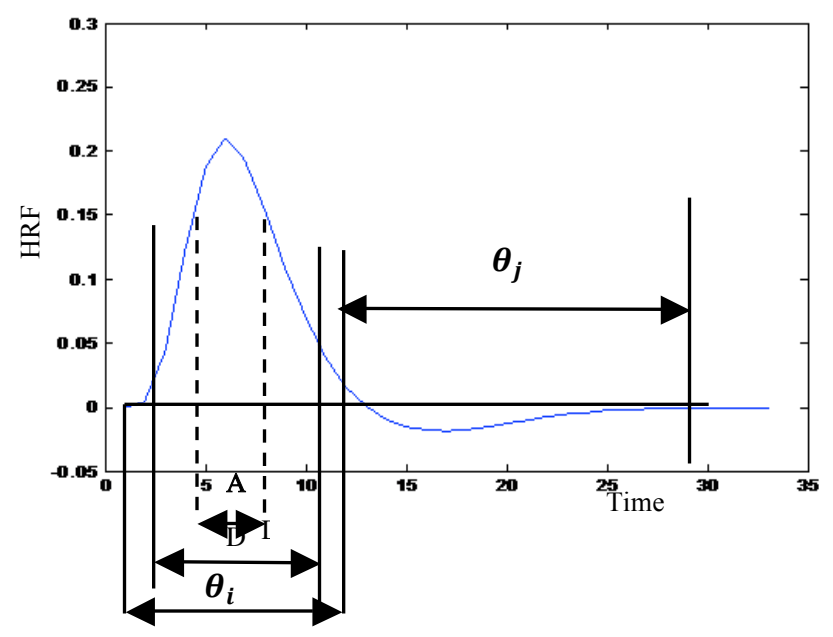

Figure 2. : the proposed model of Dempster Shafer for Canonical hemodynamic response function

To compute basic belief assignments BBA and other Dempster Shafer metrics, the $m\left(t_{1}\right)$ has been first computed using equations 5 and 6 . Then, belief measures of each modality (fMRI and EEG) have been selected. Thus, the formula described bellow has been used, it consists in the transformation of each signal $\mathrm{S}(\mathrm{t})$ of fMRI data or EEG signal into density of probability function by using the formula:

$$
\mathrm{m}(\mathrm{ti})=\frac{1}{\alpha} \int_{\mathrm{ti}-1}^{\mathrm{ti}}|\mathrm{S}(\mathrm{t})| \mathrm{dt} \text { for } \mathrm{i}=1 \text { to } \mathrm{n} \quad \mathrm{m}(\mathrm{ti})=0 \quad, \quad \mathrm{i}=0
$$

In the above equation, $\alpha$ is calculated as:

$$
\alpha=\int_{0}^{\mathrm{tn}}|\mathrm{S}(\mathrm{t})| \mathrm{dt}
$$

We obtain vectors of masses function for each modality. Then, these vectors have been employed for computing combination rule measures using the equations (3) and (4) that need to determine the intersection intervals $A=B \cap C$ which are different of empty set $\emptyset$. Figure 3 illustrates how to detect the interval A. 


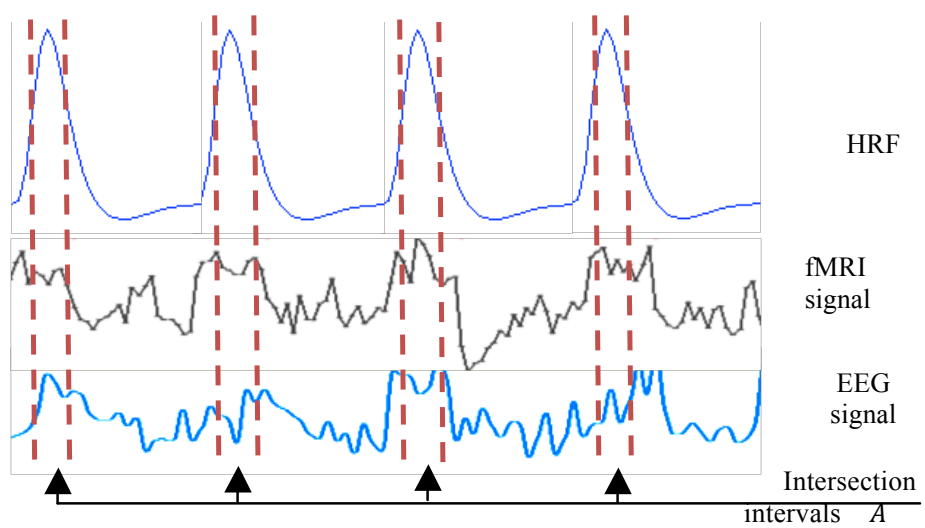

Figure 3. Figure shows how to detect the interval A

\section{Proposed Method}

As will be illustrated in Figure 4, the proposed scheme for EEG-fMRI symmetrical fusion is as follows: (i) pre-processing both fMRI and EEG data, (ii) generating HRF model by DS method, (iii) Calculating the $\mathrm{m}_{\text {мех }}()$ for each voxel, (iv) computing $\mathrm{m}_{\text {вео }}()$ after extracting ERP from EEG signals and then, $(\mathrm{v}) \mathrm{m}_{\text {мак }}()$ and $\mathrm{m}_{\text {гея }}()$ metrics have been used to compute $\mathrm{m}_{\text {мвнгево }}()$ measure. This combination rule measure $\left(\mathrm{m}_{\text {миянека }}()\right)$ has been used to extract activated voxels via clustering. To separate the activated voxels from non-activated voxels, the clustering methods have been used because they can separate data into groups based on certain similarities. In this step, the PHA has been employed. As mentioned above, PHA generates automatically a number of clusters which is less than or equal to the selected max number (C). Furthermore, among these clusters, we show the target cluster and more information like the number of voxels by cluster and the centroid of cluster denoted by (c) as well. The process is described in Dempster Shafer algorithm (algorithm DS) and consists of six steps as follows:

\section{Algorithm DS:}

- IMPUT: fMRI data, EEG signals, HRF

- OUTPUT: $\mathrm{m}_{\mathrm{fMRI}}() \quad,. \mathrm{m}_{\mathrm{EEG}}(),. \mathrm{m}_{\mathrm{EEG}, \mathrm{fMRI}}($.$) , activated areas$

- $\quad$ step 1: first model HRF by using DS method, described in section 2

- $\quad$ step 2: project HRF model with fMRI signal and compute $\mathrm{m}_{\mathrm{fMRI}}()$ vector for each voxels using equations (5) and (6).

- $\quad$ step 3: from EEG to ERP

- $\quad$ step 4: the same process applied in step 2 has been repeated on ERP to compute vector of $\mathrm{m}_{\mathrm{EEG}}()$

- $\quad$ step 5: compute the combination rule measure using the equations aforementioned in (3) and (4)

- $\quad$ step 6: use PHA method to cluster vector of $\mathrm{m}_{\mathrm{EEG}, \mathrm{fMRI}}($.$) combination measure in order to$ identify active areas . 


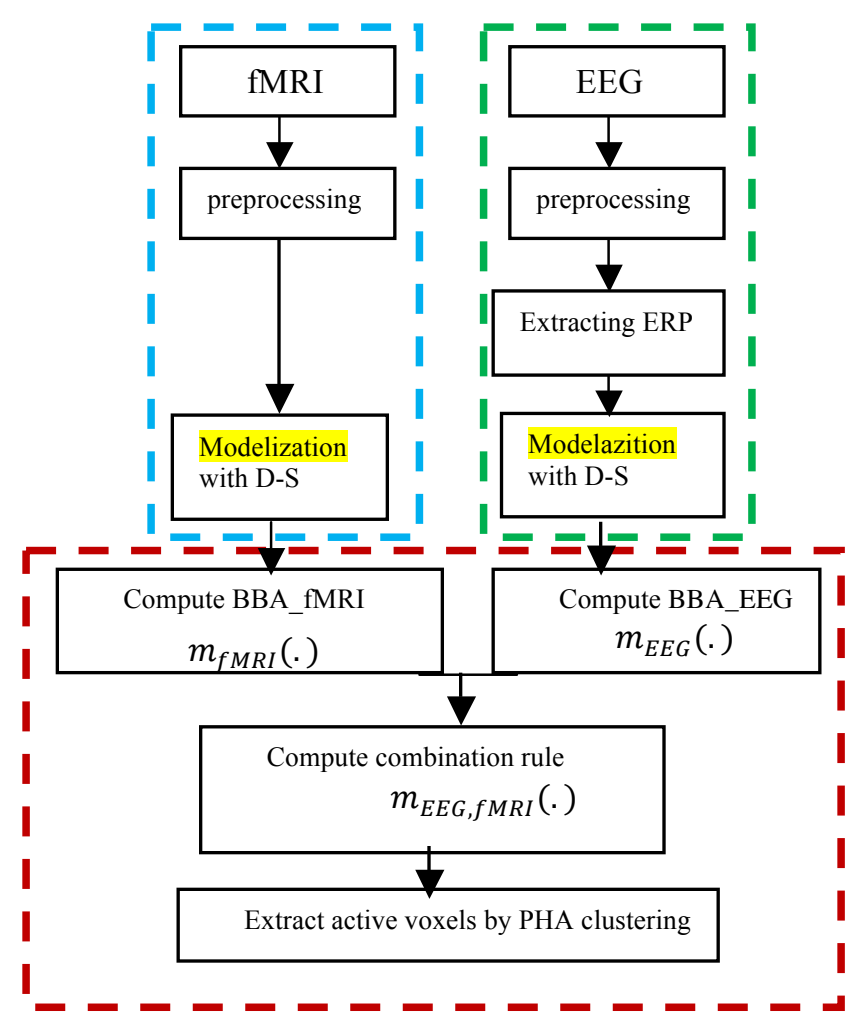

Figure 4. flowchart of the proposed method. The chart is devided into three main parts. The blue part concerns preprocess and modelisation of fMRI data.The green part is about EEG data and contains three steps; preprocessing, extracting ERP and modelisation by DS. The red part denotes clustering extract activated voxels

\section{$5 \quad$ Experiments and results}

The proposed method that relies on Dempster Shafer theory has been evaluated on both synthetic and real data. The performance of the introduced method has been based on statistical measurements. The MATLAB has been used for all implementations. At last, a comparison with jointICA has been provided to evaluate the efficiency of the proposed method. The evaluation metrics and experimental results are discussed below.

\subsection{Evaluation metrics}

This subsection describes the evaluating metrics serving to analyze the performance of the introduced approach and to establish a comparison with the jointICA. Basically, two main metrics have been given in [34]: (i) True activation rate (TAR) presents the ratio between the number of voxels correctly identified as activated and the total number of truly activated voxels and (ii) false activation rate (FAR) is known as the ratio between the number of voxels incorrectly identified as activated and the total number of truly non-activated voxels. A more comprehensive description of these two metrics allows to correctly plotting the Receiver Operating Characteristics Curve ( ROC) [35] for comparing between methods.

To compute these measures, it should be noted that jointICA has been performed on the basis of the t-test that uses a p-value or Z-threshold to map the activated voxels. The introduced method

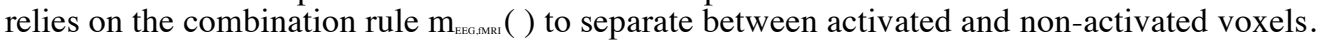




\subsection{Artificial dataset}

First, the proposed method has been tested on artificial dataset developed by Correa et al. [36] with their simulation algorithm. This dataset is available at (http://mlsp.umbc.edu/simulated_fmri_data.html). It contains eight sources where each simulated source is of $60 \times 60$ image with 100 point courses. However, in the current experiment, the virtual source for fMRI has been created into a two dimensional spatial map, as depicted in Figure 5, as the same size of the real fMRI data. Given that the information obtained from the brain is a stochastic process, a collection of sinusoid signals of the ERP data has been considered.

Let $\phi$ be the Gaussian random delay distributed with zero mean and unit variance and let $\mathrm{d}$ be the amplitude. Accordingly, ERP sources have been simulated by using the formula:

$\mathrm{x}(\mathrm{n})=100 \times \mathrm{d}^{\mathrm{n}} \sin (2 \pi \mathrm{fn}+\varphi), \quad \mathrm{n}=1 \ldots \ldots 200$

This sinusoid signal is with a mixture of very low frequencies from [0.014- $0.811 \mathrm{~Hz}$ ]
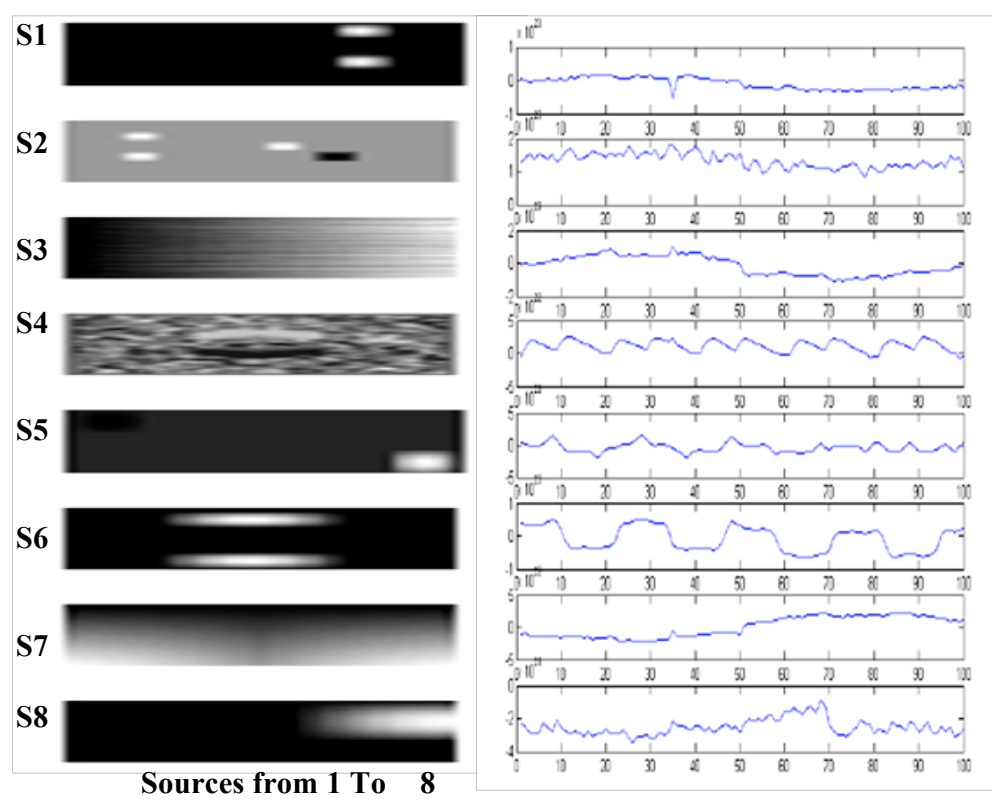

(a)
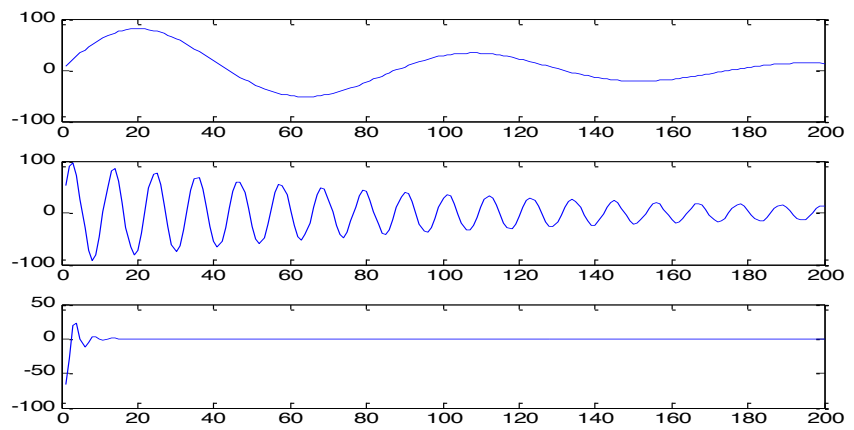

(b)

Figure 5. Artificial data taken from [36]. (a) Artificial fMRI data contains eight sources where each simulated source is of $60 \times 60$ image with 100 point courses $\quad$ (b) the selected ERP simulation generated by using( equa. 7 )

The source $S$ with a size of $(8 \times 3339)$ has been obtained by mixing the different FMRI sources. In the next step, the Demspter method has been applied on these data to get the $\mathrm{m}_{\text {мв }}(\mathrm{)})$. Then, the same algorithm has been applied on ERP dataset to compute $\mathrm{m}_{\text {гес }}()$. Once both $\mathrm{m}_{\text {во }}$ and $\mathrm{m}_{\text {мег }}$ have 


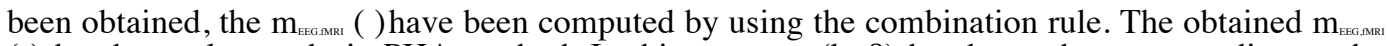
( ) has been clustered via PHA method. In this process, $(k=8)$ has been chosen according to the number of simulated data sources. The obtained results are presented in Figure 5 where different slices have been clearly shown (mixed areas) in different sources. This figure presents the mixed activated areas detected by the proposed method. As it is clear in the first four slices on the left, the activated regions are depicted. The different sources (S1, S2,S5 , S6 and part of S4) figure 6 (a) . Slices on the right show the activated areas of each source in (S4, S6 and S8). Comparing our results with those obtained with jointICA, it can be seen that jointICA detects only (S3,S4,S5,S6) sources (see Figure 6 (b)). Thus, the proposed approach has obviously shown its ability to yield a clear distinction between areas in terms of activation.
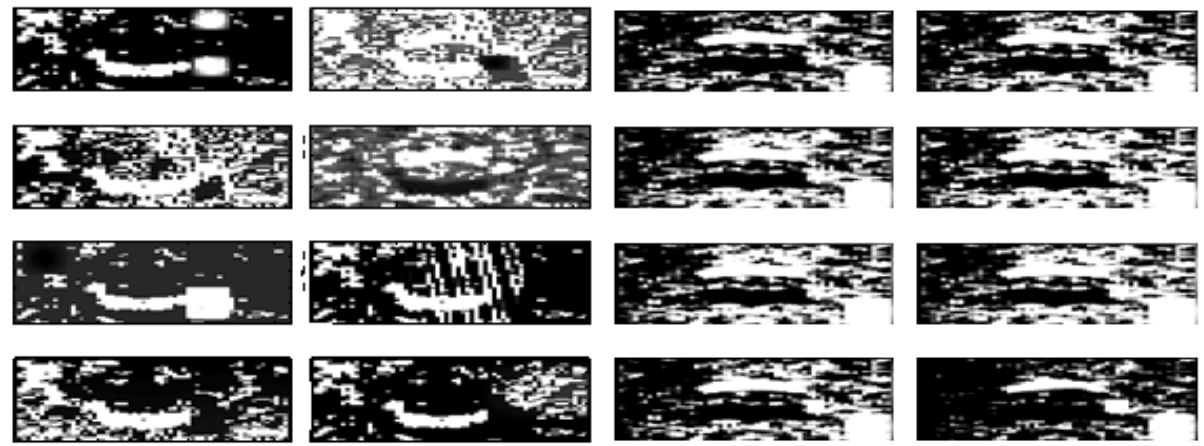

(a)
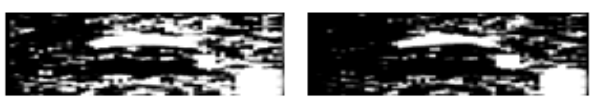

(b)

Figure 6. (a) The obtained results by the proposed method that show the mixed area in each slice (b) the JointICA results

Table1 describes the quantitative comparison between introduced method and jointICA using the true activation rate (TAR) and false activation rate (FAR). It's clear from this table that each method achieves better result more than $90.25 \%$. Also from this table the proposed method detect mor accratly than jointICA for the sources (S1,S2, S6 ,S7, S8). Then jointICA outperform the proposed method for sources $(\mathrm{S} 3, \mathrm{~S} 4, \mathrm{~S} 5)$. Table 2 show the average of measures (TAR and FAR).

Table 1 present true and false activation rates by the proposed method and jointICA

\begin{tabular}{|c|c|c|c|c|c|c|c|c|c|}
\hline \multicolumn{2}{|l|}{ source } & S1 & S2 & S3 & S4 & S5 & S6 & S7 & S8 \\
\hline \multirow{2}{*}{$\begin{array}{l}\text { Proposed } \\
\text { method }\end{array}$} & TAR\% & 97.25 & 93.50 & 98.25 & 90.75 & 98.50 & 96.75 & 92.75 & 91.75 \\
\hline & FAR & 0.0275 & 0.0650 & 0.0175 & 0.0925 & 0.0150 & 0.0325 & 0.0725 & 0.0825 \\
\hline \multirow[t]{2}{*}{ jointICA } & TAR\% & 93.50 & 91.75 & 98.50 & 93.00 & 98.75 & 94.25 & 91.25 & 90.25 \\
\hline & FAR & 0.0650 & 0.0825 & 0.0150 & 0.0700 & 0.0125 & 0.0575 & 0.0875 & 0.0975 \\
\hline
\end{tabular}

Table 2: Average of TAR and FAR of the proposed method and jointICA to the simulated dataset.

\begin{tabular}{|c|c|c|}
\cline { 2 - 3 } \multicolumn{1}{c|}{} & AVG( TAR) & AVG( FAR) \\
\hline Proposed method & $94,93 \%$ & 0.051 \\
\hline jointICA & $93.87 \%$ & 0.061 \\
\hline
\end{tabular}

From the outcome, it is very motivating to identify more activated areas. Thus, the introduced method can outperform better than the jointICA method in detecting more accurate activation for the simulated dat.

\subsection{Real data}

Besides simulation data, the current method has been evaluated also on a real fMRI and EEG dataset to access its performance. This data was described in [15], and was collected while 
participants were performing an auditory oddball. Prior to analysis, data goes through a series of preprocessing steps to identify and remove artifacts and validate model assumptions as well. Therefore, the fMRI slices require to be spatially realigned at first. However, spatial smoothing may cause unforeseen changes to occur into data. Thus, for better performance, spatial smoothing has been avoided. Then, the mean value has been subtracted from each of the time series and the variance has been normalized to a unit. The previous steps have been realized via SPM tools (Statistical Parametric Mapping).then the proposed method was applied on each voxel. In this case, the $\mathrm{m}_{\text {мв }}()$ and $\mathrm{m}_{\text {вв }}()$ was computed by using equations 5 and 6 . Then, the both modalities fMRI and EEG data was fuse by employing the combination rule in order to get avector of $\mathrm{m}_{\text {вемм }}($ ) by using equations 3 and 4 . Finally this vector is used for extracting activated voxels by using PHA clustering method. In addition we proceed to change the max number of cluster (k) by increment then we calculate the centroid (c) with the number of voxels in the target cluster. Then, the activated regions were shown. The same process has been repeated till no new cluster will be generated. Table 3 presents the results of $\mathrm{m}_{\text {вас омк }}()$ clustering by PHA method. The plots depicted in Figure 7 present the various numbers of voxels by max number of clusters.

Table 3 result of $\mathrm{m}_{\mathrm{EG}, \mathrm{arR}}()$ clustering by PHA method

\begin{tabular}{lll}
\hline $\begin{array}{c}\text { Number max of } \\
\text { cluster }(\mathbf{C})\end{array}$ & Number of voxels & Center c \\
\hline 3 & 2235 & 0.0053 \\
4 & 2184 & 0.0051 \\
5 & 2184 & 0.0051 \\
6 & 2184 & 0.0051 \\
7 & 2184 & 0.0051 \\
8 & 2184 & 0.0051 \\
9 & 1378 & 0.0062 \\
10 & 822 & 0.0069 \\
11 & 783 & 0.0068 \\
12 & 783 & 0.0068 \\
13 & 783 & 0.0068 \\
14 & 783 & 0.0068 \\
15 & 783 & 0.0068 \\
\hline
\end{tabular}

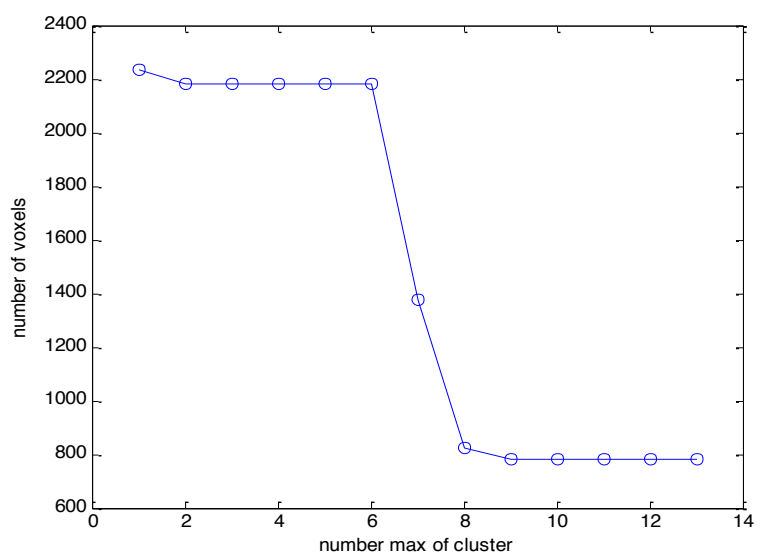

Figure 7. (a) Variation in the number of voxels by number max of clusters k.

By varying the max number $(\mathrm{k})$, false positive voxels may be obtained if $\mathrm{k}$ is small or some voxels may be neglected if $(\mathrm{k})$ is great. Thus, a threshold should be selected for better distinction of activated voxels. Thus, the average of the centroids of the target clusters has been used. By computing the average of the centroids (c), we get 0.006. Figure 8. (a) Illustrates slices that indicate activated regions while figure 8. (b) shows the activated areas for both components (red and blue). Qualitatively it is clear that introduced method detect activated region like jointICA method applied on the auditory data. In the term of quantitative comparison of the results obtained with the proposed approach and those obtained with jointICA that has been applied to analyze simultaneous EEG-fMRI data that is illustrated in Figure 9. 

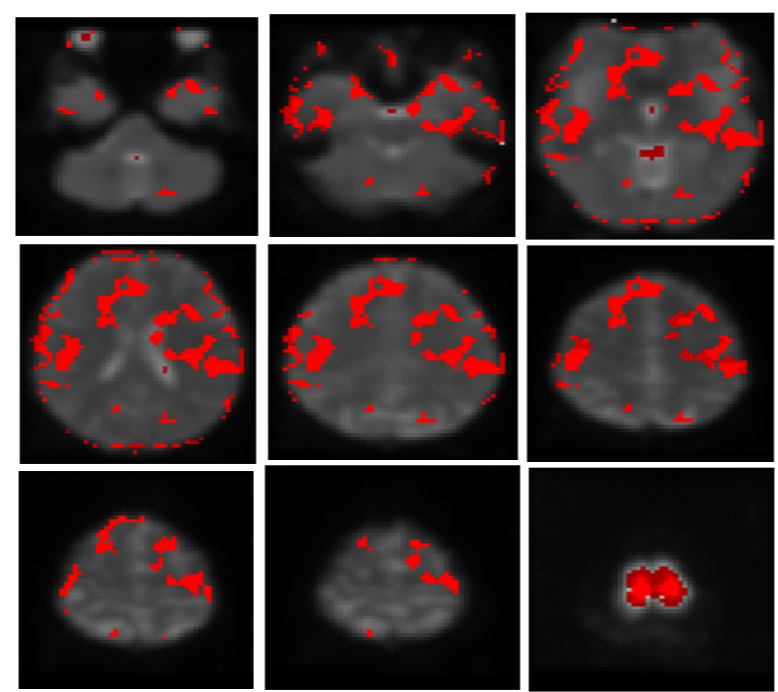

(a)

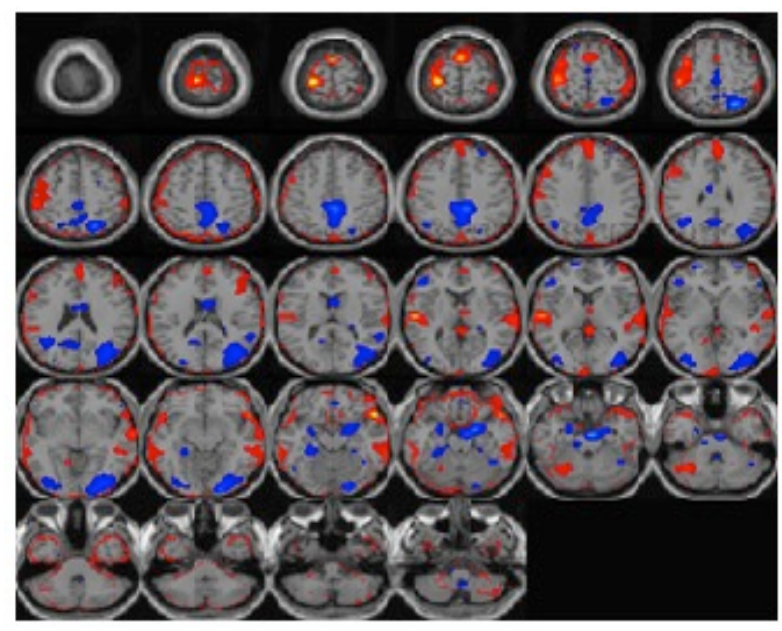

(b)

Figure 8. : (a) Activated areas in some slices by proposed method (b) Activated areas in slices by jointICA method, provided in both coulor red and blue for each compenent

As stated above in the current study, the proposed method depends on DS method that consists of two main components: The HRF model obtained by DS and $\mathrm{m}_{\text {песмег }}()$ measure. For selecting activated voxels, PHA method must be used for clustering this measure.

To validate the efficiency of the introduced approach, a comparison between the proposed algorithm and jointICA has been performed. It should be noted that jointICA is performed on the basis of the t-test that uses a p-value or Z-threshold to map the activated voxels. Given that the definition of $p$-value is similar to that of the false activated position rate in the jointICA, the $p$ value can be used as a metric for choosing thresholds. Thus, several plots have been generated for both true and false activation rates at different thresholds ( $m_{\text {ввалия }}, p$-value). Consequently, the experiment has been performed by changing thresholds for computing the metrics. Figure 9 depicts the variation of true and false activation rates according to $\mathrm{m}_{\text {ввомия }}()$ using the proposed algorithm. It seems obvious that the number of true activated voxels tends to be lower in true activation rate. However, the pace of false activation rate remains stable in the interval [0.001$0.005]$ then it gradually decreases. 


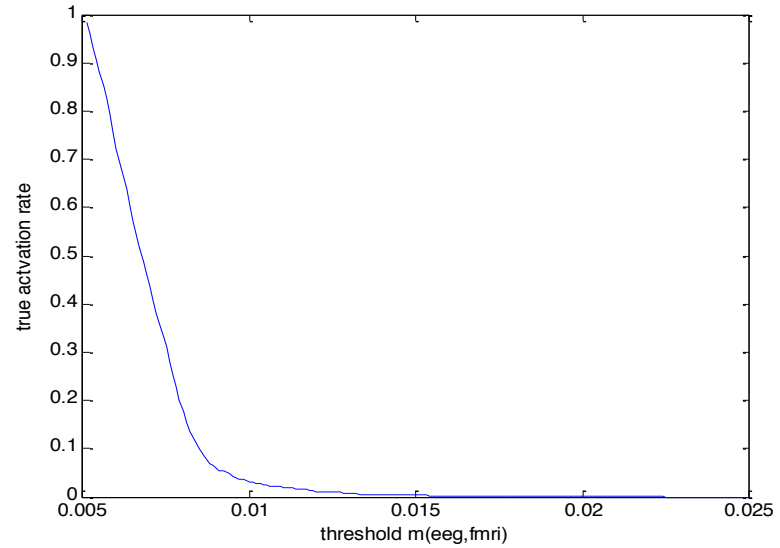

(a)

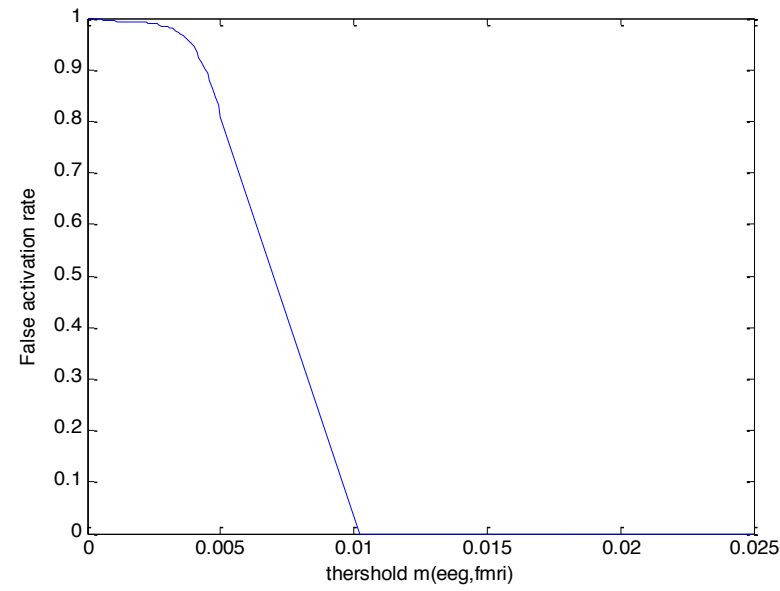

(b)

Figure 9. . Number of true positives (a) and false positives (b) using different thresholds of ( $\mathrm{m}_{\mathrm{EEG}, \mathrm{fMRI}}$ ).

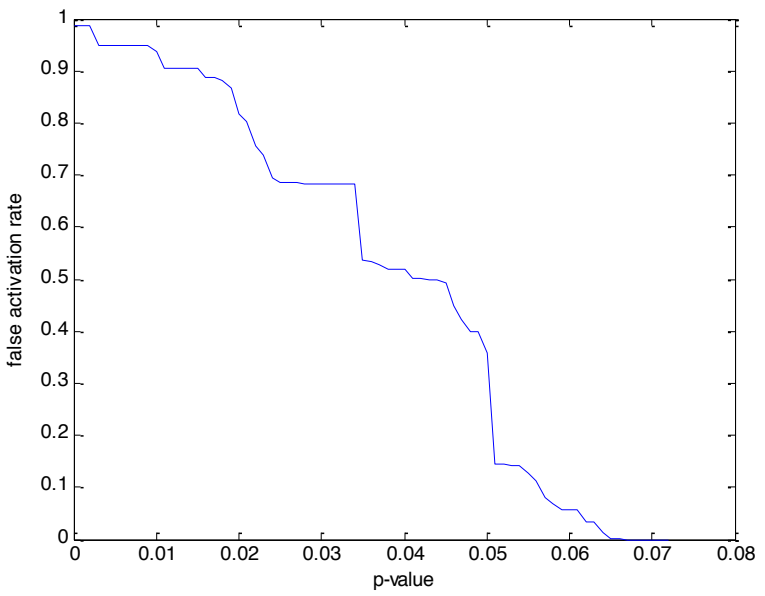

(a) 


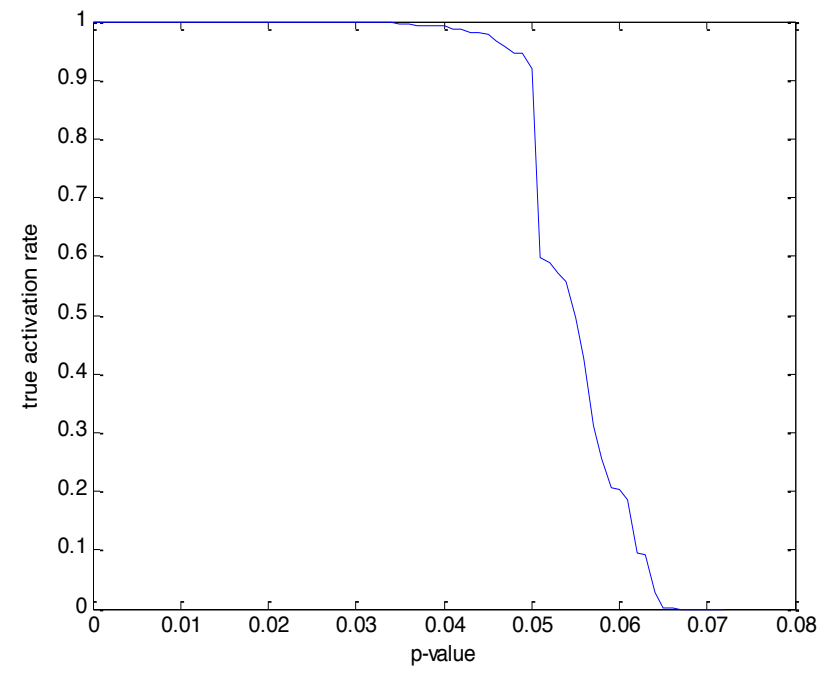

(b)

Figure 10. The number of (a) false activation rate (b) by using several p-values

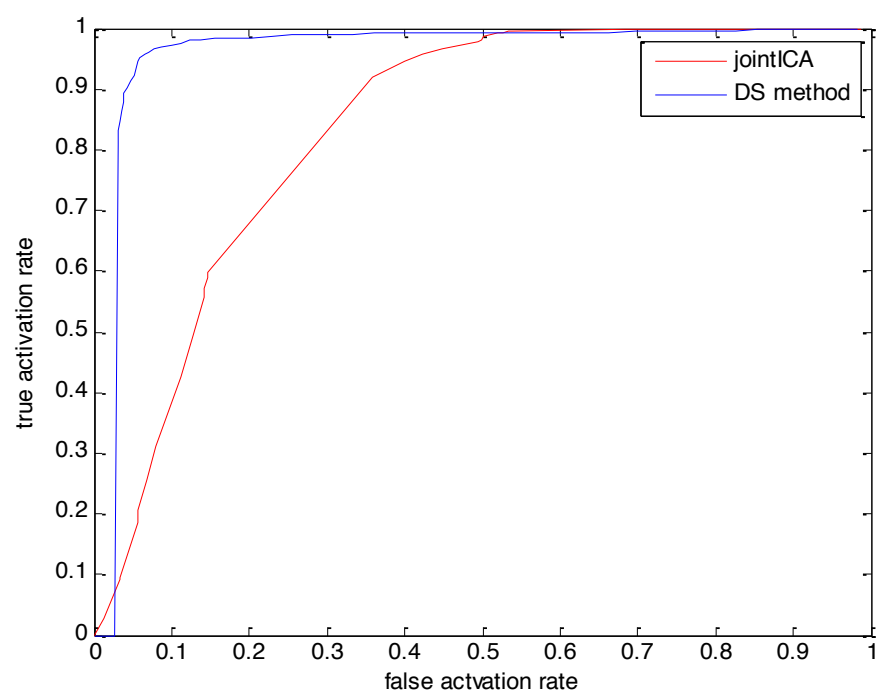

Figure 11. ROC curves for the comparison of jointICA and the introduced method

Figure 10 (a) shows that the true activation rate remains the same till the p-value reaches 0.05 when it starts to fall down gradually. Figure 10 (b) illustrates that false activation rate continues to decrease gradually when the p-value increases and sometimes it remains fixed as in] 0.025, 0.035[. We then specifically compare the obtained results with those gained by using jointICA. The ROC curves for both methods are shown in Figure 11. It is obviously observed that the ROC curve obtained by jointICA is much lower than that obtained by the proposed method. While joint-ICA provided few true positives with few false positives, the new method has detected more true positives without increasing the number of false position. However, the area under ROC curve for DS method is 0.877 and 0.733 for jointICA method. Therefore, this comparison reveals that the proposed algorithm provides a better performance in terms of identifying more true positives than jointICA. 


\section{Conclusion}

This paper introduces a new framework to identify activation areas in simultaneous EEG-fMRI data. Basically, the approach relies on Dempster Shafer theory taking into account the application of basic belief assignment and rule of combination. PHA method has been also employed to clustering the obtained measures for separate activate voxels from non-activated ones. An extensive performance analysis has been provided with both artificial and real datasets. Compared to joint ICA, the obtained activation map confirmed the efficiency of the proposed method for EEG-fMRI fusion in yielding a clear distinction between activated and non-activated areas. Although the focus has been placed on the analysis of auditory data, the introduced approach can be extended to explore various aspects of brain activity and to detect brain illnesses such as epilepsy.

\section{References}

1 Zhang, Y., Wang, Z., \& Hu, Z., Nonlinear electroencephalogram analysis of neural mass model, Journal of Medical Imaging and Health Informatics, 5(4), 783-788. (2015).

2 Kim, D. H., Lee, J. J., Han, B. S., \& You, J. H. ,Cortical or Subcortical Neural Networks During Dynamic Neuromuscular Core Stabilization: A fMRI Blood Oxygen-Level Dependent (BOLD) Analysis., Journal of Medical Imaging and Health Informatics, 6(7), 1732-1734 (2016).

3 3Mahjoory, K., Nikulin, V. V., Botrel, L., Linkenkaer-Hansen, K., Fato, M. M., \& Haufe, S. ,Consistency of EEG source localization and connectivity estimates, bioRxiv, 071597. (2016).

4 Ives JR, Warach S, Schmitt F, Edelman RR, Schomer DL, Monitoring the patients EEG during echo planar MRI. Electroencephalography and Clinical Neurophysiology, 87: 417-420. (1993)

5 Pflieger ME, Greenblatt RE. Nonlinear analysis of multimodal dynamic brain imaging data. Int J Bioelectromagnetism; 3(2001)

6 Xu Lei, Chuan Qiu, Peng Xu, Dezhong Yao. A parallel framework for simultaneous EEG-fMRI analysis methodology and simulation. Neuroimage; 52:1123-1134 (2010)

7 Martinez Montes E, Valdes Sosa PA, Miwakeichi F, Goldman RI, Cohen MS. Concurrent EEG-fMRI analysis by multiway partial least squares. Neuroimage; 22: 1023-1034. (2004)

8 Eichele T, Debener S, Calhoun VD, Specht K, Engel AK, Hugdahl K, von Cramon DY, Ullsperger M. Prediction of human errors by maladaptive changes in event-related brain networks. Proc Natl Acad Sci USA ;18 March 2008; USA : PNAS. pp. 6173-6178. (2008)

9 Benar CG, Grova C, Kobayashi E, Bagshaw AP, Aghakhani Y, Dubeau F, Gotman J. EEG-fMRI of epileptic spikes concordance with EEG source localization and intracranial EEG. Neuroimage; 30: 1161-1170. (2006)

10 Daunizeau J, Grova C, Marrelec G, Mattout J, Jbabdi S, Pélégrini Issac M, Lina, JM, Benali H. Symmetrical event related EEG-fMRI information fusion in a variational Bayesian framework. Neuroimage; $36: 69-87$. (2007)

11 Valdes Sosa PA, Sanchez Bornot JM, Sotero RC, Iturria Medina Y, Aleman-Gomez Y, Bosch-Bayard J, Carbonell F, Ozaki T. Model driven EEG-fMRI fusion of brain oscillations. Hum Brain Mapp; 30: 2701-2721. (2009)

12 Xu Lei , Pedro A. Valdes Sosa, Dezhong Ya. EEG-fMRI fusion based on independent component analysis integration of data-driven and model-driven methods. J integr neurosci; 11: 313-337 (2012)

13 Munck J C, Goncalves S I, Huijboom L, Kuijer J P, Pouwels P J, Heethaar RM, Lopes da Silva FH. The hemodynamic response of the alpha rhythm an EEG-fMRI study. Neuroimage; 35: 1142-1151. (2007)

14 Eichele T, Calhoun V D, Debener S. Mining EEG-fMRI using independent component analysis. Int J Psychophysiol; 73: 53-61 (2009)

15 Calhoun V D, Adali T, Giuliani N R, Pekar J J, Kiehl1 KA, Pearlson G D. Method for multimodal analysis of independent source differences in schizophrenia combining gray matter structural and auditory oddball functional data. Hum Brain mapp; 27: 47-62. (2006)

16 Calhoun V D, Liu J, Adali T. A review of group ICA for fMRI data and ICA for joint inference of imaging genetic and ERP data. Neuroimage; 45:163-172. (2009)

17 Eichele T, Calhoun V D, Moosmann M, Specht K, Jongsma, M L, Quiroga R Q, Nordby H, Hugdahl K. Unmixing concurrent EEG-fMRI with parallel independent component analysis. Int J Psychophysiol; 67:222-234. (2008)

18 Mijovic B, Vanderperren K, Novitskiy N, Vanrumste B, Stiers P, Van den Bergh B, Lagae L, Sunaert $S$, Wagemans J, Van Huffel S, De Vos M. The why and how of JointICA results from a visual detection task. Neuroimage ; 60: 1171-1185. (2012)

19 Mijovic B, De Vos M, Vanderperren K, Machilsen B, Sunaert S, Van Huffel S, Wagemans J. The dynamics of contour integration a simultaneous EEG-fMRI study. Neuroimage, 88: 10-21. (2014)

20 Poznanski RR, Riera JJ. fMRI models of dendritic and astrocytic networks. J Integr Neurosci ; 5: 273-326. (2006)

21 Kiebel S J, Garrido M I, Friston K J. Dynamic causal modelling of evoked responses the role of intrinsic connections. Neuroimage; 36: 332-345. (2007) 
22 Chen C C, Kiebel SJ, Kilner JM, Ward N S, Stephan KE, Wang W J, Friston K J. A dynamic causal model for evoked and induced responses. Neuroimage; 59: 340-348. (2012)

23 Gotman J. Epileptic networks studied with EEG-fMRI. Epilepsia; 49: 3, 42-51. (2008)

24 Sturzbecher M J, Tedeschi W, Cabella B C, Baa O, Neves U P, de Araujo D B. Non-extensive entropy and the extraction of BOLD spatial information in eventrelated functional MRI. phys med biol; 54: 161-174. (2009)

25 Sato J R, Rondinoni C, Sturzbecher M, de Araujo D B, Amaro E Jr. From EEG to BOLD brain mapping and estimating transfer functions in simultaneous EEG-fMRI acquisitions. Neuroimage; 50: 1416-1426. (2010)

26 Friston KJ. Analysis of functional MRI time series. Hum Brain Mapp 1994; 1: 153-171.

27 27Lu Y, Wan Y. PHA a fast potential-based hierarchical agglomerative clustering method. Pattern recogn; 46: 1227-1239. (2013)

28 Dempster AP. Upper and lower probabilities induced by a multivalued mapping. Ann Math Statist; 38 : 325-339. (1967)

29 Liu, J., Lu, X., Li, Y., Chen, X., \& Deng, Y., A new method based on Dempster-Shafer theory and fuzzy c-means for brain MRI segmentation, Measurement Science and Technology, 26(10), 105402. . (2015)

30 Cui, H., Peng, L., Song, H., Wang, G., Li, J., Guo, L., \& Yuan, C.. Dempster-Shafer multifeature fusion for pedestrian detection. Advances in Mechanical Engineering. (2015)

31 Shafer G. A Mathematical Theory of Evidence. Princeton University Press, Princeton NJ, (1976).

32 Yager R R. Dempster shafer belief structures with interval valued focal weights. Int J intell syst; 16 : 497-512. (2001)

33 33Aguirre GK, Zarahn E, Desposito M. The variability of human BOLD hemodynamic responses. Neuroimage; 8: 360-369. (1998)

34 Francois G Meyer, Jatuporn Chinrungrueng. Spatiotemporal clustering of fMRI time series in the spectral domain. Med image anal; 9: 51-68. (2005)

35 35Sorenson J, Wang X. ROC methods for evaluation of fMRI techniques. MRM; 36: 737-744. (1996)

36 36Correa N, Li Y O, Adali T , Calhoun V. Comparison of blind source separation algorithms for fMRI using a new Matlab toolbox GIFT. In: IEEE International Conference on Acoustics Speech Signal Processing (ICASSP); 23-23 March 2005; Philadelphia: IEEE. pp. 401-403.( 2005) 